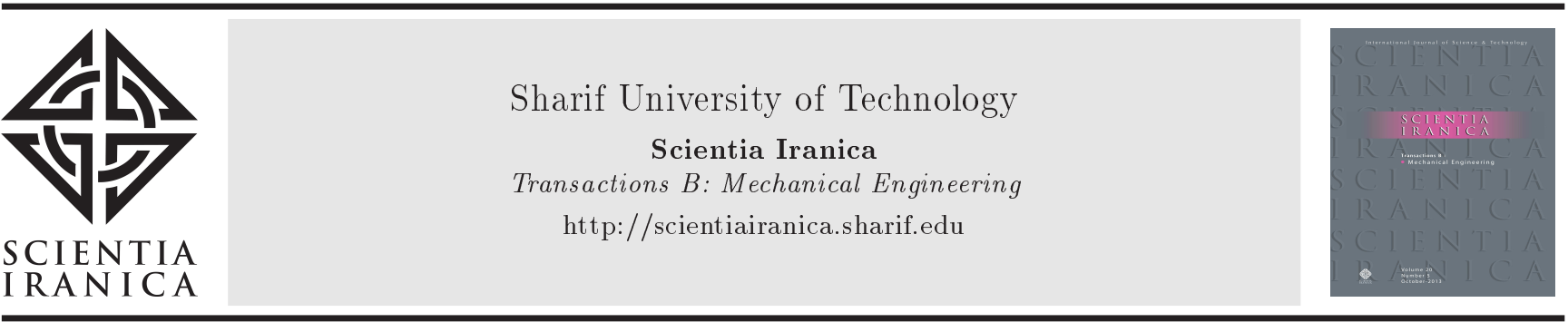

\title{
Sagittal range of motion of the thoracic spine using standing digital radiography: A throughout comparison with non-radiographic data reviewed from the literature
}

\author{
S.S. Madinei and N. Arjmand* \\ Department of Mechanical Engineering, Sharif University of Technology, Tehran, P.O. Box 11155-9567, Iran.
}

Received 12 December 2016; received in revised form 13 August 2017; accepted 14 May 2018

\author{
KEYWORDS \\ Thoracic spine; \\ Range of motion; \\ Kyphosis (Cobb) \\ angle; \\ Digital radiography; \\ Imaging; \\ Biomechanical \\ modelling.
}

\begin{abstract}
Previous studies have measured thoracic Range of Motion (RoM) using either skin-mounted devices or supine CT-imaging and have reported on quite different RoMs. Given the inherent shortcomings of measurements of vertebrae movements from the overlying skin, the present study aims to measure normal RoM of the thoracic spine in the sagittal plane using the upright digital radiography. Lateral radiographs of the thoracic spine were obtained from eight asymptomatic male subjects in upright standing and full forward flexion using a mobile U-arm digital radiographic system. Total (T1T12), upper (T1-T6), and lower (T6-T12) thoracic RoMs were measured. A throughout comparison of available skin-based measurements in the literature was carried out. Mean of total (T1-T12) thoracic RoM was $22.5^{\circ}$ (SD $4.1^{\circ}$ ), most of which was generated by the lower (T6-T12) thoracic spine as compared to upper (T1-T6) thoracic spine $\left(15.5^{\circ}\right.$ versus $\left.7.1^{\circ}, p<0.001\right)$. These RoMs were within the lower range of the data previously reported by other skin-based approaches. While skin-based measurements suffer from the inter sensor-skin-vertebra movements and supine imaging techniques do not allow maximal trunk flexion, standing radiography remains as the gold-standard technique. Evaluation of thoracic spine RoM has implications in both patient discrimination for diagnosis and in biomechanical models for estimation of spinal loads.
\end{abstract}

(C) 2019 Sharif University of Technology. All rights reserved.

\section{Introduction}

Thoracic spine pain is considered as a growing workrelated disease $[1,2]$. An epidemiological investigation of a large population of workers in France showed that one fifth of female and one tenth of male workers sustained thoracic pain [3]. Results of a survey conducted in Japan revealed that surgeries on the thoracic spine accounted for $\sim 11 \%$ of all spinal surgeries [4]. Due to

\footnotetext{
*. Corresponding author. Tel.: +982166165684;

Fax: +982166000021

E-mail address: arjmand@sharif.edu (N. Arjmand)
}

doi: $10.24200 /$ sci. 2018.20503 effect of the musculoskeletal disorders and pain on joint movements, evaluation of the sagittal Range of Motion (RoM) of the thoracic spine (i.e., maximum relative vertebral rotation of $\mathrm{T} 1$ to $\mathrm{T} 12$ ) can be used as a tool to discriminate between patients and healthy individuals, subsequent diagnostic purposes, and manual therapy treatments of individuals suffering from shoulder outlet impingement syndrome [5]. Moreover, quantification of the thoracic spine RoM is important in the musculoskeletal models to estimate spinal loads and, thus, design effective prevention (ergonomics) programs [6-8].

There are three approaches to measuring the sagittal RoM of the thoracic spine. The most common technique is through skin-surface sensors or markers such as marker-camera [9], electronic inclinome- 
ter [10,11], electromagnetic [12,13], and inertial [14] sensors. Such studies suffer from the unavoidable movements between skin and vertebra as well as sensor and skin. The second technique involves the use of in vitro cadaveric specimens [15] in which the stabilizing role of some bony passive (e.g., sternum and rib cage) and active (muscles) tissues is excluded, thus resulting in an overestimation of thoracic RoM [14]. The last approach involves medical imaging such as Computed Tomography (CT) [16]. The latter study does not suffer from the foregoing shortcomings; however, it has an important limitation, i.e., full flexion RoM is not reached as subjects must keep a supine posture during the test.

The above-mentioned studies have measured considerably different sagittal T1-T12 RoMs for the thoracic spine varying from $\sim 18$ to $33^{\circ}$ for uprightforward flexion $[9,10,12-14,17]$ and from $\sim 32$ to $70^{\circ}$ for total flexion-extension [11,13,15-17]. Although these differences partly result from dissimilarities in subjects' characteristics (e.g., age and gender), different techniques/devices used may also play a role. Moreover, there has been controversy over the contribution of the upper and lower regions of the thoracic spine in generating the total T1-T12 RoM. While our recent study using an inertial tracking device [14], as well as two other skin-based measurements [13,15], indicates that most of the thoracic RoM is produced by the relative rotation of the lower thoracic vertebrae, the CT imaging technique [16] indicates relatively larger RoM at T1-T3 levels as compared to the lower thoracic levels.

A gold-standard database according to which findings of the existing skin-based measurements for thoracic RoM can be verified is missing in the literature. Imaging machines, which provide measurements in the standing posture such as digital radiology, are the gold-standard devices for evaluation of the spinal RoMs. This is because such measurements are based on in vivo images acquired from the vertebra itself rather than the skin surface. The present study, hence, aims to measure sagittal RoMs of the lower (T6-T12), upper (T1-T6), and total (T1-T12) thoracic spine in asymptomatic subjects (from relaxed upright to full voluntary forward flexion) using digital radiographic imaging. A throughout comparison of the measurements with the previously reported data using non-radiographic approaches was also carried out. Furthermore, the T1-T12 thoracic kyphosis (Cobb) angle was evaluated in the upright posture, and its correlation with the thoracic RoM was investigated.

\section{Materials and methods}

\subsection{Radiographic system}

Lateral radiographs of the thoracic spine were obtained using a mobile U-arm digital radiographic system
(Sedecal ${ }^{\circledR}$, X Plus LP Plus, Spain) with a flat-panel detector incorporating $43 \mathrm{~cm} \times 43 \mathrm{~cm}$ field of view (Figure 1).

\subsection{Subjects and protocol}

Eight asymptomatic non-scoliosis male subjects $(28.5$ years old (SD 5.7), $176.7 \mathrm{~cm}$ (SD 9.1), and $78.2 \mathrm{~kg}$ (SD 12.9)) with no history of spinal surgery or recent musculoskeletal pain volunteered for the measurement sessions. Each subject, after being familiarized with the imaging process including the radiation exposure risk, signed an informed consent form. Proper approval to acquire radiographs was acquired from our ethics committee. Volunteers were requested to stand in their neutral upright posture within the U-arm close to the detector with the source and detector in the mediallateral direction (Figure 1). Vertical travel and sourceto-image distances were adjusted for each subject based on his body height to have the best field of view. To allow clear visualization of the thoracic spine in upright posture, arms and shoulders were extended forward. After acquiring an image in this posture, subjects were requested to perform maximal voluntary forward flexion with knee extended. The detector height was adjusted to allow the flexed thoracic spine to remain in the field of view of the machine, and a second image was acquired. A third image of the whole cervical spine was also obtained to further help an experienced radiologist identify the $\mathrm{T} 1$ vertebra in the images.

\subsection{Data analysis}

The image DICOM (Digital Imaging and Communications in Medicine) files were imported into Mimics ${ }^{\circledR}$ (version 17.0, Materialise, Leuven, Belgium) to digitally measure thoracic RoMs and upright T1-T12 kyphosis angle on a personal computer. A previously-

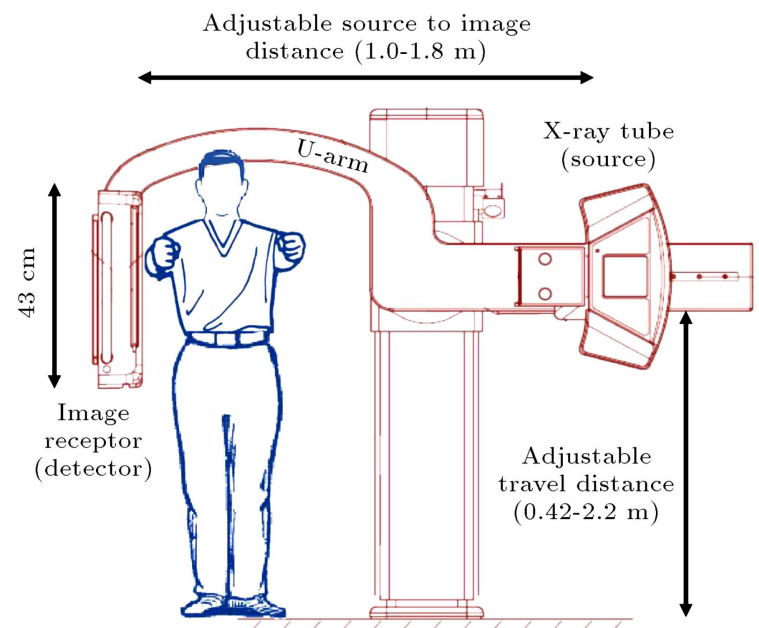

Figure 1. A schematic presentation of the U-arm digital radiographic system as well as subject standing in upright posture close to the flat panel (detector) with arms and shoulders extended forward. 


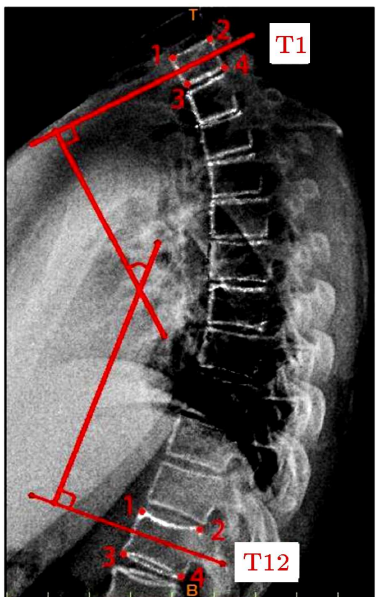

(a)

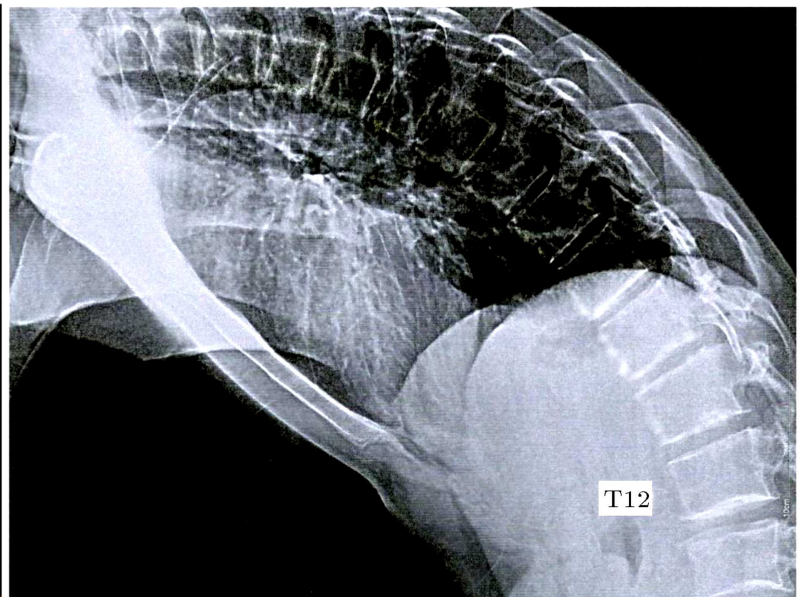

(b)

Figure 2. (a) Identification of the $\mathrm{T} 1$ and $\mathrm{T} 12$ ventral/dorsal corners and their midpoints to measure mid-planes of each vertebra on lateral radiographs in the upright posture. (b) A typical radiograph of the thoracic spine in the flexed posture.

developed approach to measuring sagittal rotation of the lumbar vertebrae from lateral radiographs $[18,19]$ was used here for the thoracic spine. This method has been described to be independent of distortion, axial rotation, or lateral tilt of the film or vertebral body as well as to produce minimal inter- and intra-observer variabilities $[18,19]$. In brief, ventral and dorsal corners of T1, T6, and T12 vertebrae (as landmarks of maximal distance from the center of area of the vertebral body) were identified in both upright and fully flexed postures (Figure 2). Ventral and dorsal midpoints between corners 1 and 3 and corners 2 and 4 were subsequently pinpointed, thus allowing for the identification of the mid-plane of each vertebra (Figure 2). Variation of the angle between T1 and T12 mid-planes as participants flexed forward from their relaxed upright to maximal flexion posture was defined as the total thoracic RoM. The upper (T1-T6) and lower (T6-T12) thoracic RoMs were measured similarly. Moreover, the global T1T12 Cobb angle (thoracic kyphosis) was measured as the angle formed by the line attaching the two upper corners of $\mathrm{T} 1$ (along the upper endplate of T1) and the line attaching the two lower corners of T12 (along the lower endplate of T12). The Cobb angle approach is described to be the most common [20] and gold-standard [21] method for radiographic analysis of the spinal curvatures. When required and before the aforementioned measurements, the Windowing tool of Mimics ${ }^{\circledR}$ was used to enhance the contrast of the vertebrae on the digital radiographic images (Figure 2). All measurements were performed by two trained raters (blind to each other).

\subsection{Statistical analyses}

The Kolmogorov-Smirnov test was used to test normality on the T1-T12 RoM and T1-T12 Cobb angle. The Intra-class Correlation Coefficients (ICC) were calcu- lated to assess inter-rater reliability. Paired t-tests were conducted to compare lower (T1-T6) and upper (T6T12) RoMs. Spearman's rank correlation coefficient was used to measure rank correlation between the thoracic RoM and Cobb angle.

\section{Results}

For two subjects, all measurements were performed based on $\mathrm{T} 2$ vertebra, as image clarity at the T1 level was limited due to the overlying osseous structures. The data distribution was found normal. Intraclass Correlation Coefficient (ICC) analysis indicated an excellent inter-rater reliability for both T1-T12 RoM $(\mathrm{ICC}=0.88)$ and Cobb angle $(\mathrm{ICC}=0.96)($ Table 1$)$. The mean of total (T1-T12) thoracic RoM was $22.5^{\circ}$ $\left(\mathrm{SD} 4.1^{\circ}\right.$ ) (ranged from 17.3 to $29.3^{\circ}$ ), of which $15.5^{\circ}$ (SD $3.1^{\circ}$ ) was generated by the relative flexion of $\mathrm{T} 6$ to T12 (i.e., T6-T12 RoM) and the remaining (7.1 ${ }^{\circ}$ SD $1.8^{\circ}$ ) by the relative flexion of $\mathrm{T} 1$ to $\mathrm{T} 6$ (i.e., T1-T6 RoM) (Table 1). The measured sagittal T1-T12 RoM was in the lower range of non-radiographic measurement data (Figure 3). For all subjects, lower thoracic (T6-T12) made a greater contribution to produce the total thoracic (T1-T12) RoM as compared with the upper thoracic spine (T1-T6) $(p-$ value $<0.001)$. The upright T1-T12 Cobb angle ranged from $\sim 28$ to $45^{\circ}\left(38.7^{\circ}, \mathrm{SD} 6.1^{\circ}\right)$ and fell also within the lower normal range of the reported data in the literature (Figure 4). A non-significant negative correlation was found between the T1-T12 Cobb angle and T1-T12 $\operatorname{RoM}(r=-0.48, p-$ value $=0.233)$.

\section{Discussion}

This study aimed to measure sagittal RoM of the thoracic spine, whose magnitude based on the skin- 
Table 1. Upper (T1-T6), lower (T6-T12), and total (T1-T12) thoracic Range of Motions (RoM) from upright to full flexion as well as upright T1-T12 kyphosis (Cobb angle) for the eight subjects participated in the study. Measurements of both raters as well as their mean values are reported.

\begin{tabular}{|c|c|c|c|c|}
\hline \multirow{3}{*}{ Subject } & \multicolumn{4}{|c|}{ Rater 1} \\
\hline & \multicolumn{3}{|c|}{ RoM (deg) } & \multirow{2}{*}{$\begin{array}{c}\text { Cobb (deg) } \\
\text { T1-T12 }\end{array}$} \\
\hline & T1-T6 & T6-T12 & T1-T12 & \\
\hline 1 & 6.8 & 12.5 & 19.3 & 32.7 \\
\hline 2 & 6.0 & 12.9 & 18.9 & 45.0 \\
\hline 3 & 6.5 & 18.7 & 25.1 & 31.4 \\
\hline $4^{*}$ & 7.4 & 20.2 & 27.7 & 30.1 \\
\hline $5^{*}$ & 6.3 & 16.1 & 22.4 & 42.9 \\
\hline 6 & 6.1 & 12.5 & 18.6 & 42.5 \\
\hline 7 & 6.9 & 15.4 & 22.3 & 40.6 \\
\hline 8 & 12.1 & 15.2 & 27.3 & 42.0 \\
\hline Mean & 7.3 & 15.4 & 22.7 & 38.4 \\
\hline \multirow[t]{2}{*}{$(\mathrm{SD})$} & $(2.0)$ & $(2.9)$ & $(3.7)$ & $(5.9)$ \\
\hline & \multicolumn{4}{|c|}{ Rater 2} \\
\hline 1 & 5.8 & 13.8 & 19.6 & 33.4 \\
\hline 2 & 5.7 & 13.8 & 19.5 & 42.5 \\
\hline 3 & 5.3 & 19.3 & 24.6 & 35.3 \\
\hline $4^{*}$ & 5.9 & 18.3 & 24.2 & 27.0 \\
\hline $5^{*}$ & 7.9 & 17.3 & 25.2 & 42.2 \\
\hline 6 & 5.9 & 10.1 & 15.9 & 46.1 \\
\hline 7 & 7.6 & 11.0 & 18.6 & 41.7 \\
\hline 8 & 10.7 & 20.6 & 31.4 & 43.2 \\
\hline \multirow{3}{*}{$\begin{array}{c}\text { Mean } \\
(\mathrm{SD})\end{array}$} & 6.9 & 15.5 & 22.4 & 38.9 \\
\hline & $(1.8)$ & $(3.9)$ & $(4.9)$ & $(6.4)$ \\
\hline & \multicolumn{4}{|c|}{ Mean of raters } \\
\hline 1 & 6.3 & 13.2 & 19.5 & 33.0 \\
\hline 2 & 5.8 & 13.4 & 19.2 & 43.8 \\
\hline 3 & 5.9 & 19.0 & 24.9 & 33.3 \\
\hline $4^{*}$ & 6.7 & 19.3 & 25.9 & 28.5 \\
\hline $5^{*}$ & 7.1 & 16.7 & 23.8 & 42.6 \\
\hline 6 & 6.0 & 11.3 & 17.3 & 44.3 \\
\hline 7 & 7.3 & 13.2 & 20.5 & 41.1 \\
\hline 8 & 11.4 & 17.9 & 29.3 & 42.6 \\
\hline Mean & 7.1 & 15.5 & 22.5 & 38.7 \\
\hline$(\mathrm{SD})$ & $(1.8)$ & $(3.1)$ & $(4.1)$ & $(6.1)$ \\
\hline
\end{tabular}

*: For two subjects, all measurements were performed

based on the $\mathrm{T} 2$ vertebra as image clarity at the $\mathrm{T} 1$ level was limited.

based measurements has been somewhat contentious in eight asymptomatic individuals using, for the first time, the gold-standard upright radiographic images. Mean upright to full flexion RoM of the thoracic spine was $22.5^{\circ}\left(\mathrm{SD} 4.1^{\circ}\right)$, of which $\sim 60 \%$ was provided by the relative flexion of $\mathrm{T} 6$ to $\mathrm{T} 12$ (lower thoracic). A throughout review of the literature revealed that the existing non-radiographic (skin-based) measurements generally reported larger thoracic RoMs. Our measurements for the thoracic T1-T12 Cobb angle (38.7 $(\mathrm{SD}$ $\left.6.1^{\circ}\right)$ ) fell within the lower range of other radiographic and skin-based measurements. The measured T1T12 RoM and thoracic kyphosis had a non-significant negative correlation.

\subsection{Limitations}

The study had some limitations that should be considered when interpreting the findings. First, analysis of radiographic images suffered from low-clarity images. For two subjects, we could not properly locate the $\mathrm{T} 1$ vertebra due to the overlying bony structures; measurements were thus carried out using the T2 vertebra. Second, due to the invasive nature of the study (risk of radiations), we were obliged by our ethics committee to minimize the number of subjects (agreed on total of 8 subjects), especially that we had to take one additional image (apart from the two images taken from the thoracic spine in upright and flexed postures needed for the measurement of RoM) from the cervical spine to allow for the identification of T1 vertebra on the image (total of 3 images for each subject). The likely effect of gender and age could not, therefore, be investigated in the present study. This could have also adversely compromised the power of our statistical analyses. For this reason, a non-parametric correlation test (Spearman's rank correlation) rather than a common Pearson's correlation analysis was conducted to measure rank correlation between the thoracic RoM and Cobb angle. Third, although the Cobb method is the most frequent approach to the evaluation of the spinal curves [22-31] and is also widely recognized as the gold-standard approach in clinical applications $[20,21,32]$, some have questioned its validity due to inherent errors in identification of the vertebral mid-plane slopes and in using 2D measurements rather than 3D ones [24]. Other methods for radiologic assessment of the spinal curvatures have been suggested [33]; however, the Cobb angle remains the clinical standard technique [20].

\subsection{Comparison of thoracic RoM with non-radiographic approaches}

Few studies have investigated sagittal thoracic RoM. We are unaware of any standing radiographic (or other imaging) assessment of the thoracic RoM. A throughout review of the literature revealed that previous studies used skin-based tools [9-14,17], cadaveric specimens [15], and CT images in the supine posture [16] to measure thoracic RoM. When comparing the findings of different works for thoracic RoM, one should consider dissimilarities between the methodologies used 


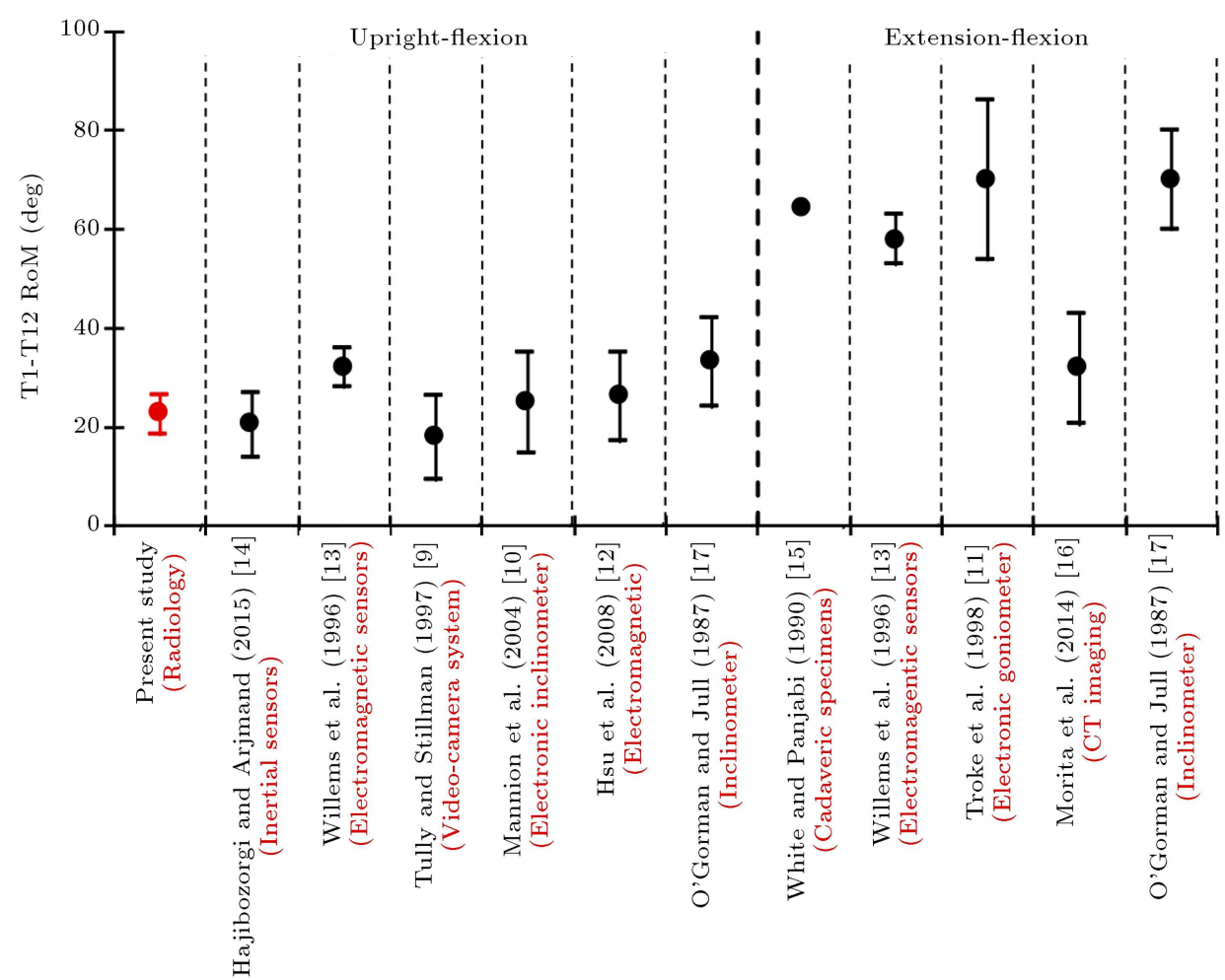

Figure 3. Comparison of the mean ( $\pm 1 \mathrm{SD}$ ) values (in degrees) of thoracic (T1-T12) Range of Motion (RoM) (either from the upright standing posture to full flexion (upright-flexion) or from full extension to full flexion (extension-flexion)) measured in the current study with those reported in the literature (equipment used are also indicated). For O'Gorman and Jull (1987) [17], RoM data of young individuals (22-29 years old) are presented.

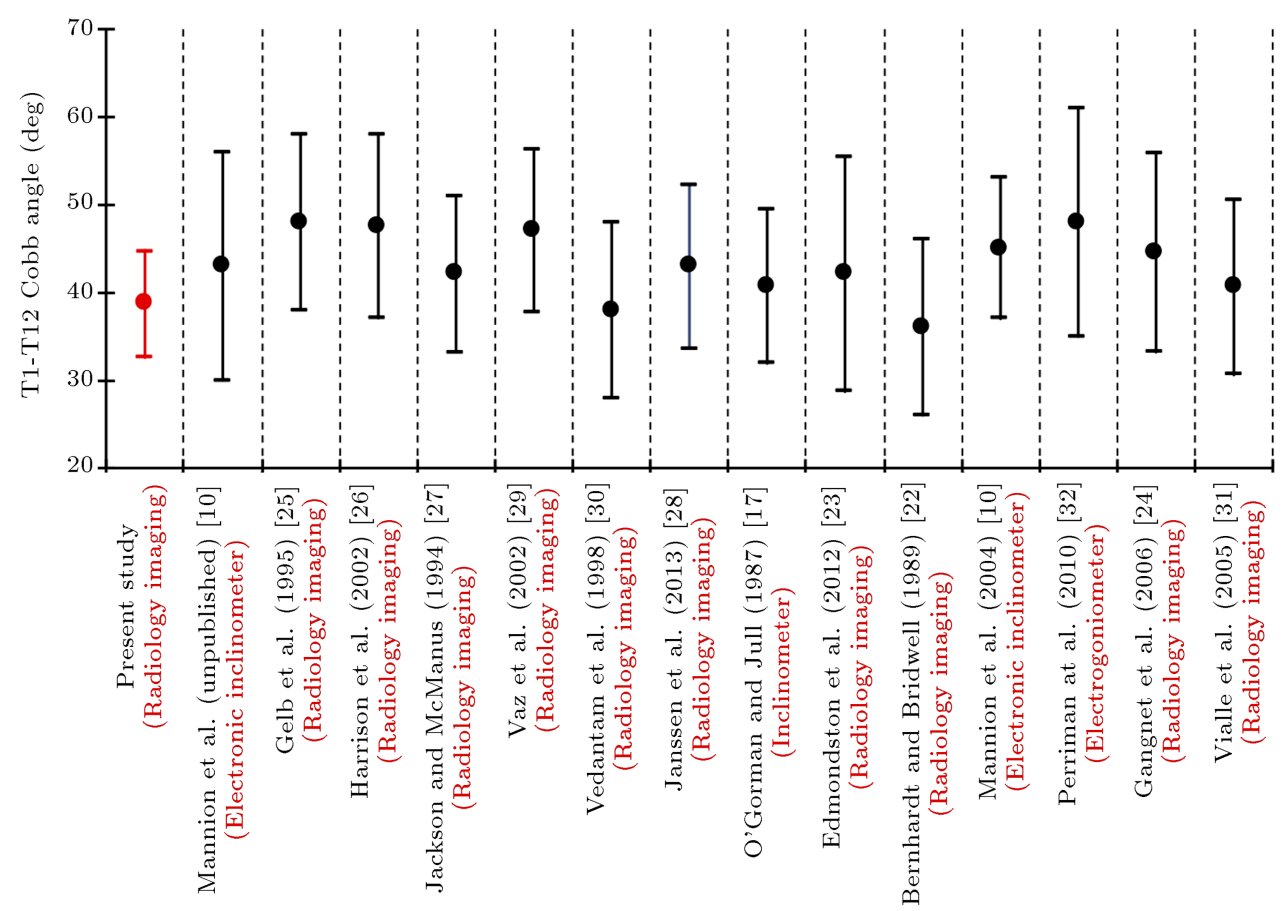

Figure 4. Comparison of the mean $( \pm 1 \mathrm{SD})$ values (in degrees) of full thoracic (T1-T12) upright Cobb angle measured in the current study with those reported in the literature (equipment used is also indicated). Data of Vedantam et al. (1998) [30] and Bernhardt and Bridwell (1989) [22] are based on T3-T12 Cobb angle, data of Edmondston et al. (2012) [23] based on T3-T11 Cobb angle, and data of Janssen et al. (2013) [28] and Vialle et al. (2005) [31] based on T4-T12 Cobb angle. 
(e.g., in vivo versus in vitro or inertial sensors versus inclinometer devices), subjects' characteristics, as well as the posture under which RoMs are measured (e.g., standing versus supine or standing-flexion RoM versus full extension-flexion RoM). Moreover, differences between findings of the previous in vivo investigations can be partly due to the lack of a common standard upright or full flexion posture. For instance, while we asked participants to flex forward to reach their maximal flexion RoM, Troke et al. (1998) [11] asked subjects to flex forward so as to look back through their legs. This could partly explain why they measured relatively larger thoracic RoM (full extension-flexion RoM of $70^{\circ}$ (SD 16.2 $\left.{ }^{\circ}\right)$ ), while Tully and Stillman (1997) [9] who asked subjects to only touch their toes measured the smallest RoM (upright to flexion RoM of $17.8^{\circ}$ (SD $\left.8.6^{\circ}\right)$ ). Finally, it is to be noted that such comparisons should not be considered as validation or reliability of the methodology used. For the sake of validation of skin-based approaches, one should measure and compare thoracic RoM on the same subjects using both the skin-based and imaging approaches. As for the reliability of skin-based measurements, one should use different measurement techniques to evaluate RoM on the same subjects [34].

Apart from our recent study that measured the sagittal RoM of the thoracic spine in forty healthy young males using inertial sensors [14], there are five more studies in the literature that reported on upright to full flexion thoracic RoM (Figure 3). Our present measurements for the thoracic RoM ranged from 17.3 to $29.3^{\circ}\left(22.5^{\circ}\left(\mathrm{SD} 4.1^{\circ}\right)\right)$ (Table 1) and agreed closely with our recent measurements using inertial sensors $\left(20.5^{\circ}\left(\mathrm{SD} 6.5^{\circ}\right)\right)$. The measured RoM also fell within the lower range of measurements by other skin-mounted devices (Figure 3 ). The only work that reported smaller RoM as compared to our present and previous [14] investigations was that of Tully and Stillman (1997) [9] $\left(17.8^{\circ},\left(\mathrm{SD} 8.6^{\circ}\right)\right)$ in which full flexion posture was the toe-touching posture. There were three studies that reported only full extensionflexion thoracic RoM $[11,15,16]$; thus, their data cannot be directly compared with the present measurements. Nevertheless, full flexion-extension thoracic RoM measured in the supine posture using CT images $\left(31.7^{\circ}\right.$ $\left(\mathrm{SD} 11.3^{\circ}\right)$ ) [16] was considerably smaller than and in disagreement with values reported by others $(\sim$ $\left.58-70^{\circ}\right)[11,13,15,17]$ as maximal trunk flexion cannot be reached in the supine posture.

In agreement with the only in vitro investigation [15] and two skin-based measurements using electromagnetic [13] and inertial [14] tracking devices, yet in disagreement with the $\mathrm{CT}$ imaging investigation in supine posture [16], our findings showed that lower thoracic spine (T6-T12) made a greater contribution to producing the total thoracic (T1-T12) RoM as compared with the upper thoracic spine (T1-T6) $\left(15.5^{\circ}\right.$

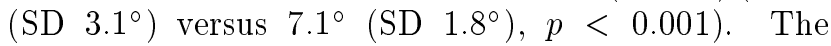
in vitro study [15], however, reported relatively larger RoM for the lower thoracic spine, i.e., $\sim 12^{\circ}$ of full flexion-extension RoM for T11-T12 alone that could be due to the fact that the some stabilizing bony structures (sternum and rib cage) and muscles were removed from their cadaveric specimens. Invasive insertion of Kirschner wires into T11 and T12 spinous processes [35] was used to measure a full in vivo T11T12 flexion-extension RoM of only $2.7^{\circ}$ that further confirms an overestimation of RoM in the cadaveric specimens.

\subsection{Comparison of thoracic kyphosis with some (selected) literature}

Unlike RoM, normal thoracic kyphosis has been extensively measured using both radiographic and skinbased methods [10,17,22-32], and a wide range of data have been reported (Figure 4 ). The normal thoracic kyphosis is accepted in the range of 20 to $50^{\circ}$ [22]. Our measurements for the thoracic kyphosis (T1-T12 Cobb angle) ranged from $\sim 28$ to $45^{\circ}$ (Table 1 ) $\left(38.7^{\circ}\left(\mathrm{SD} 6.1^{\circ}\right)\right)$ that fell within the lower range of other measurements (Figure 4); there were only two works that reported smaller thoracic kyphosis $[22,30]$; however, both reported T3-T12 Cobb angle rather than the T1-T12 kyphosis. Our relatively smaller Cobb angle might be partly explained by the young age of our subjects as aging causes a considerable increase in the thoracic kyphosis [17]. In a large population study on 670 young individuals (5-20 years old), the mean of T2-T12 RoM was measured to be $37.6^{\circ}$ (i.e., smaller than that measured here) [36]; according to some investigations, the normal upper limit of T1T12 kyphosis was $40^{\circ}[37,38]$. It is also important to note that our valid range of data for the upright $\mathrm{T} 1$ T12 thoracic kyphosis assured our proper calculation methodology for the thoracic RoM. This is because the measured thoracic RoM is actually equal to the difference between thoracic kyphosis (Cobb angles) in upright and full flexion postures.

\subsection{Applications in biomechanical models}

Apart from its clinical importance, evaluation of thoracic RoM is also essential in the musculoskeletal models of the spine for estimation of force in muscles and loads on spine joints [6-8]. Based on the fact that flexion RoM of the thoracic spine is relatively smaller than RoM of the lumbar (reported to be $\sim 52^{\circ}$ in a popular radiographic investigation [39]), musculoskeletal models generally assume that the whole thorax moves as a single rigid body. The role of ligamentous passive tissues of the thorax in balancing gravity and moments is therefore overlooked in these models. Our recent modeling study [40] indicated that a thoracolumbar 
musculoskeletal model with a rigid thorax predicted slightly or moderately lower compressive loading (18 to $22 \%$ depending on the simulated task) than a flexiblethorax model. According to the present findings, biomechanical models should, therefore, account for $\sim 22^{\circ}$ of total T1-T12 flexion in full forward flexion activities. The T1-T12 flexion angle increases almost linearly (with trunk flexion) as individuals flex forward from the upright posture [14].

\section{Conclusion}

Evaluation of RoM of the thoracic spine has applications in both clinical and biomechanical investigations. For the first time, standing radiographic measurements of sagittal RoM of the thoracic spine were performed on healthy individuals. A throughout comparison between our gold-standard radiographic data and those measured by non-radiographic (skin-based) approaches reviewed from the literature was carried out. Our measured T1-T12 RoM of the thoracic spine from upright to full voluntary flexion $\left(22.5^{\circ}\right.$ (SD 4.1 $\left.{ }^{\circ}\right)$ ) were in the lower range of the skin-based measurements and agreed well with our recent measurements $\left(20.5^{\circ}\right.$ (SD 6.5०)) using inertial sensors [14]. Upright T1T12 thoracic kyphosis was also measured $\left(38.7^{\circ}\right.$ (SD $\left.6.1^{\circ}\right)$ ) and compared with some selected radiographic and skin-based data from the literature. This valid range of data for the upright thoracic kyphosis further confirms the validity of our measured thoracic RoM that is equal to the difference between the thoracic kyphosis in upright posture and that in full flexion posture.

\section{Acknowledgment}

This work was supported by grants from Sharif University of Technology (Tehran, Iran). The authors appreciate assistance of Dr. K. Abdollahifard in acquiring radiographic images and identifying $\mathrm{T} 1$ and $\mathrm{T} 12$ vertebrae on radiographs as well as Prof. M. Parnianpour and Prof. A. Shirazi-Adl for data interpretation.

\section{References}

1. Briggs, A.M., Bragge, P., Smith, A.J., Govil, D., and Straker, L.M. "Prevalence and associated factors for thoracic spine pain in the adult working population: a literature review", Journal of Occupational Health, 51(3), pp. 177-192 (2009).

2. Fouquet, N., Bodin, J., Descatha, A., Petit, A., Ramond-Roquin, A., Ha, C., and Roquelaure, Y. "0188 thoracic spinal pain prevalence in the musculoskeletal disorders surveillance network of the French Pays de la Loire region", Occupational and Environmental Medicine, 71(Suppl 1), pp. A24-A24 (2014).
3. Fouquet, N., Bodin, J., Descatha, A., Petit, A., Ramond, A., Ha, C., and Roquelaure, Y. "Prevalence of thoracic spine pain in a surveillance network", Occupational Medicine, 65(2), pp. 122-125 (2015).

4. Nohara, Y., Taneichi, H., Ueyama, K., Kawahara, N., Shiba, K., Tokuhashil, Y., Tani, T., Nakahara, S., and lida, T. "Nationwide survey on complications of spine surgery in Japan", Journal of Orthopaedic Science, 9(5), pp. 424-433 (2004).

5. Theisen, C., van Wagensveld, A., Timmesfeld, N., Efe, T., Heyse, T.J., Fuchs-Winkelmann, S., and Schofer, M.D. "Co-occurrence of outlet impingement syndrome of the shoulder and restricted range of motion in the thoracic spine - a prospective study with ultrasoundbased motion analysis", BMC Musculoskeletal Disorders, 11 (1), p. 1 (2010).

6. Arjmand, N. and Shirazi-Adl, A. "Model and in vivo studies on human trunk load partitioning and stability in isometric forward flexions", Journal of Biomechanics, 39(3), pp. 510-521 (2006).

7. Arjmand, N., Plamondon, A., Shirazi-Adl, A., Lariviere, C., and Parnianpour, M. "Predictive equations to estimate spinal loads in symmetric lifting tasks", Journal of Biomechanics, 44(1), pp. 84-91 (2011).

8. Arjmand, N., Plamondon, A., Shirazi-Adl, A., Parnianpour, M., and Larivière, C. "Predictive equations for lumbar spine loads in load-dependent asymmetric one-and two-handed lifting activities", Clinical Biomechanics, 27(6), pp. 537-544 (2012).

9. Tully, E.A. and Stillman, B.C. "Computer-aided video analysis of vertebrofemoral motion during toe touching in healthy subjects", Archives of Physical Medicine and Rehabilitation, 78(7), pp. 759-766 (1997).

10. Mannion, A.F., Knecht, K., Balaban, G., Dvorak, J., and Grob, D. "A new skin-surface device for measuring the curvature and global and segmental ranges of motion of the spine: reliability of measurements and comparison with data reviewed from the literature", European Spine Journal, 13(2), pp. 122-136 (2004).

11. Troke, M., Moore, A.P., and Cheek, E. "Reliability of the OSI CA 6000 spine motion analyzer with a new skin fixation system when used on the thoracic spine", Manual Therapy, 3(1), pp. 27-33 (1998).

12. Hsu, C.J., Chang, Y.W., Chou, W.Y., Chiou, C.P., Chang, W.N., and Wong, C.Y. "Measurement of spinal range of motion in healthy individuals using an electromagnetic tracking device", Journal of Neurosurgery: Spine, 8(2), pp. 135-142 (2008).

13. Willems, J.M., Jull, G.A., and Ng, J.F. "An in vivo study of the primary and coupled rotations of the thoracic spine", Clinical Biomechanics, 11(6), pp. 311316 (1996).

14. Hajibozorgi, M. and Arjmand, N. "Sagittal range of motion of the thoracic spine using inertial tracking device and effect of measurement errors on model predictions", Journal of Biomechanics, 49(6), pp. 913918 (2016). 
15. White III, A.A., Panjabi, M.M., Clinical Biomechanics of the Spine, 2nd Ed. pp. 102-103, Lippincott, Philadelphia, US (1990).

16. Morita, D., Yukawa, Y., Nakashima, H., Ito, K., Yoshida, G., Machino, M., Kanbara, S., Iwase, T., and Kato, F. "Range of motion of thoracic spine in sagittal plane", European Spine Journal, 23(3), pp. 673-678 (2014).

17. O'gorman, H. and Jull, G. "Thoracic kyphosis and mobility: the effect of age", Physiotherapy Practice, 3(4), pp. 154-162 (1987).

18. Brinckmann, P., Frobin, W., Biggemann, M., Hilweg, D., Seidel, S., Burton, K., Tillotson, M., Sandover, J., Atha, J., Quinnell, R., and Chiropractic, A.E.C. "Quantification of overload injuries to thoracolumbar vertebrae and discs in persons exposed to heavy physical exertions or vibration at the work-place: the shape of vertebrae and intervertebral discs-study of a young, healthy population and a middle-aged control group", Clinical Biomechanics, 9, pp. S3-S83 (1994).

19. Frobin, W., Brinckmann, P., Leivseth, G., Biggemann, M., and Reikerås, O. "Precision measurement of segmental motion from flexion-extension radiographs of the lumbar spine", Clinical Biomechanics, 11(8), pp. 457-465 (1996).

20. Briggs, A.M., Wrigley, T.V., Tully, E.A., Adams, P.E., Greig, A.M., and Bennell, K.L. "Radiographic measures of thoracic kyphosis in osteoporosis: Cobb and vertebral centroid angles", Skeletal Radiology, 36(8), pp. 761-767 (2007).

21. Harrison, D.E., Harrison, D.D., Cailliet, R., Janik, T.J., and Holland, B. "Radiographic analysis of lumbar lordosis: centroid, Cobb, TRALL, and Harrison posterior tangent methods", Spine, 26(11), pp. e235-e242 (2001).

22. Bernhardt, M. and Bridwell, K.H. "Segmental analysis of the sagittal plane alignment of the normal thoracic and lumbar spines and thoracolumbar junction", Spine, 14(7), pp. 717-721 (1989).

23. Edmondston, S.J., Christensen, M.M., Keller, S., Steigen, L.B., and Barclay, L. "Functional radiographic analysis of thoracic spine extension motion in asymptomatic men", Journal of Manipulative and Physiological Therapeutics, 35(3), pp. 203-208 (2012).

24. Gangnet, N., Dumas, R., Pomero, V., Mitulescu, A., Skalli, W., and Vital, J.M. "Three-dimensional spinal and pelvic alignment in an asymptomatic population", Spine, 31(15), pp. E507-E512 (2006).

25. Gelb, D.E., Lenke, L.G., Bridwell, K.H., Blanke, K. and McEnery, K.W. "An analysis of sagittal spinal alignment in 100 asymptomatic middle and older aged volunteers", Spine, 20(12), pp. 1351-1358 (1995).

26. Harrison, D.E., Cailliet, R., Harrison, D.D., and Janik, T.J. "How do anterior/posterior translations of the thoracic cage affect the sagittal lumbar spine, pelvic tilt, and thoracic kyphosis?", European Spine Journal, 11(3), pp. 287-293 (2002).
27. Jackson, R.P. and McManus, A.C. "Radiographic analysis of sagittal plane alignment and balance in standing volunteers and patients with low back pain matched for age, sex, and size: A prospective controlled clinical study", Spine, 19(14), pp. 1611-1618 (1994).

28. Janssen, M.M., Vincken, K.L., van Raak, S.M., Vrtovec, T., Kemp, B., Viergever, M.A., Bartels, L.W., and Castelein, R.M. "Sagittal spinal profile and spinopelvic balance in parents of scoliotic children", The Spine Journal, 13(12), pp. 1789-1800 (2013).

29. Vaz, G., Roussouly, P., Berthonnaud, E., and Dimnet, J. "Sagittal morphology and equilibrium of pelvis and spine", European Spine Journal, 11(1), pp. 80-87 (2002).

30. Vedantam, R., Lenke, L.G., Keeney, J.A., and Bridwell, K.H. "Comparison of standing sagittal spinal alignment in asymptomatic adolescents and adults", Spine, 23(2), pp. 211-215 (1998).

31. Vialle, R., Levassor, N., Rillardon, L., Templier, A., Skalli, W., and Guigui, P. "Radiographic analysis of the sagittal alignment and balance of the spine in asymptomatic subjects", The Journal of Bone \& Joint Surgery, 87(2), pp. 260-267 (2005).

32. Perriman, D.M., Scarvell, J.M., Hughes, A.R., Ashman, B., Lueck, C.J., and Smith, P.N. "Validation of the flexible electrogoniometer for measuring thoracic kyphosis", Spine, 35(14), pp. E633-E640 (2010).

33. Goh, S., Price, R.I., Leedman, P.J., and Singer, K.P. "A comparison of three methods for measuring thoracic kyphosis: implications for clinical studies", Rheumatology, 39(3), pp. 310-315 (2000).

34. Johnson, K.D., Kim, K.M., Yu, B.K., Saliba, S.A., and Grindstaff, T.L. "Reliability of thoracic spine rotation range-of-motion measurements in healthy adults", Journal of Athletic Training, 47(1), pp. 52-60 (2012).

35. Gercek, E., Hartmann, F., Kuhn, S., Degreif, J., Rommens, P.M., and Rudig, L. "Dynamic angular three-dimensional measurement of multisegmental thoracolumbar motion in vivo", Spine, 33(21), pp. 23262333 (2008).

36. Voutsinas, S.A. and MacEwen, G.D. "Sagittal profiles of the spine", Clinical Orthopaedics and Related Research, 210, pp. 235-242 (1986).

37. Bradford, D.S., Moe, J.H., and Winter, R.B. "Kyphosis and postural roundback deformity in children and adolescents", Minnesota Medicine, 56(2), pp. 114-120 (1973).

38. Fon, G.T., Pitt, M.J., and Thies Jr, A.C. "Thoracic kyphosis: range in normal subjects", American Journal of Roentgenology, 134(5), pp. 979-983 (1980).

39. Pearcy, M., Portek, I., and Shephard, J. "Threedimensional x-ray analysis of normal movement in the lumbar spine", Spine, 9(3), pp. 294-297 (1984). 
40. Ignasiak, D., Ferguson, S.J., and Arjmand, N. "A rigid thorax assumption affects model loading predictions at the upper but not lower lumbar levels", Journal of Biomechanics, 49(13), pp. 3074-3078 (2016).

\section{Biographies}

Seyed Saman Madinei received his BSc degree in Mechanical Engineering from Sharif University of Technology, Iran in 2015. He is currently a master's student in Industrial Engineering, Ergonomics at West Virginia University, USA. His field of research is occupational biomechanics and industrial ergonomics.

Navid Arjmand received his PhD degree in Mechanical Engineering from École Polytechnique de Montreal, Canada, in 2007. He is currently an Associate Professor at the Department of Mechanical Engineering at Sharif University of Technology, Tehran, Iran. His field of research is spine biomechanics (in vivo and modeling approaches). 